\title{
Rapid left atrial appendage thrombus formation before suture ligation with LARIAT
}

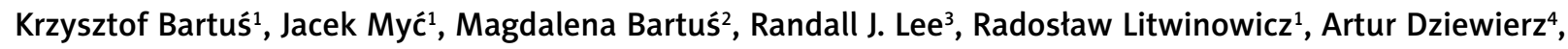 \\ Bogusław Kapelak ${ }^{1}$
}

\begin{abstract}
${ }^{1}$ Department of Cardiovascular Surgery and Transplantology, Jagiellonian University, John Paul II Hospital, Krakow, Poland 2Department of Experimental Pharmacology, Jagiellonian University, John Paul II Hospital, Krakow, Poland

${ }^{3}$ Cardiovascular Research Institute, Department of Medicine, Institute for Regeneration Medicine, University of California San Francisco, San Francisco, CA, USA

${ }^{4} 2^{\text {nd }}$ Department of Cardiology, Jagiellonian University Medical College, Krakow, Poland
\end{abstract}

Adv Interv Cardiol 2018; 14, 4 (54): 435-437

DOI: https://doi.org/10.5114/aic.2018.79876

A 74-year-old man with a history of long-lasting persistent atrial fibrillation (AF) on chronic dabigatran treatment $(2 \times 150 \mathrm{mg} /$ day $)$ was referred for left atrial appendage (LAA) closure following a severe ischemic stroke 2 years before. The patient was at high thromboembolic risk with a $\mathrm{CHA}_{2} \mathrm{DS}_{2}$-VASc score of 7 points (1 point for congestive heart failure, age, arterial hypertension, diabetes mellitus, peripheral vascular disease and 2 points for the previous stroke) and HAS-BLED of 3 points (1 point for hypertension, stroke and elderly). On admission, 2 days before the planned procedure, ECG showed sinus rhythm. Computed tomography confirmed favorable anatomy for epicardial LAA closure (Figure $1 \mathrm{~A}$ ). Dabigatran was discontinued 3 days before the procedure, and low-molecular-weight heparin was started $(2 \times 80 \mathrm{mg} /$ day, the last dose on the day of the procedure).

Before starting anesthesia at 3.57 PM the patient was confirmed with no thrombus in LAA by transesophageal echocardiography (TEE) (Figure 1 B). At that time, the patient started to experience AF. Just before starting the LAA epicardial exclusion at 4:27 PM TEE was repeated. Fresh thrombus formation in LAA was observed (Figure $1 \mathrm{C}$ ). At 4:31 PM even more solid thrombus was noted (Figure $1 \mathrm{D}$ ). The patient was excluded from LAA closure. A high-dose bolus of unfractionated heparin $(10,000 \mathrm{U})$ was given, followed by infusion of $2000 \mathrm{U} / \mathrm{h}$ with a hope to resolve the thrombus by the next day. Next day at 10.30 AM another TEE was performed and partial dilution of the thrombus was confirmed (Figure $1 \mathrm{E}$ ). No stroke, transient ischemic attack, or embolism was noted. Due to the high risk of thromboembolic events, the patient was discharged home with low-molecular-weight heparin $(2 \times 80 \mathrm{mg} /$ day $)$ and dabigatran $(2 \times 150 \mathrm{mg} /$ day $)$. After 2 months, TEE presented no signs of thrombus in the LAA. The patient underwent uncomplicated closed-chested LAA ligation with the LARIAT suture delivery device (SentreHEART, Inc., Redwood City, CA). Complete LAA closure was confirmed with TEE and contrast fluoroscopy. The patient did well after the procedure and was discharged on aspirin (150 mg/day) without any additional oral anticoagulation treatment. A follow-up TEE performed at 3 months revealed a closed LAA with no leaks. After 3 years, the patient was on no anti-platelet or anti-thrombin medications with no signs of recurrent thromboembolic complications.

The LAA is a source of more than $90 \%$ of thrombus in $A F$ and changes in cardiac rhythm from sinus rhythm to $A F$ may particularly increase the risk of thrombus formation in LAA. Oral anticoagulation is recommended as a standard treatment to prevent thromboembolic events in AF patients. However, in patients who are at high risk for thrombus formation, new therapeutic options might be considered. One of them is LAA closure, which has become an alternative treatment for AF patients [1, 2]. One possible option is percutaneous epicardial LAA closure using the LARIAT system, which was shown to be effective in thromboembolism risk reduction, even in high-risk patients [3-5].

This case report describes rapid thrombus formation in LAA despite recommended oral anticoagulant treat- 

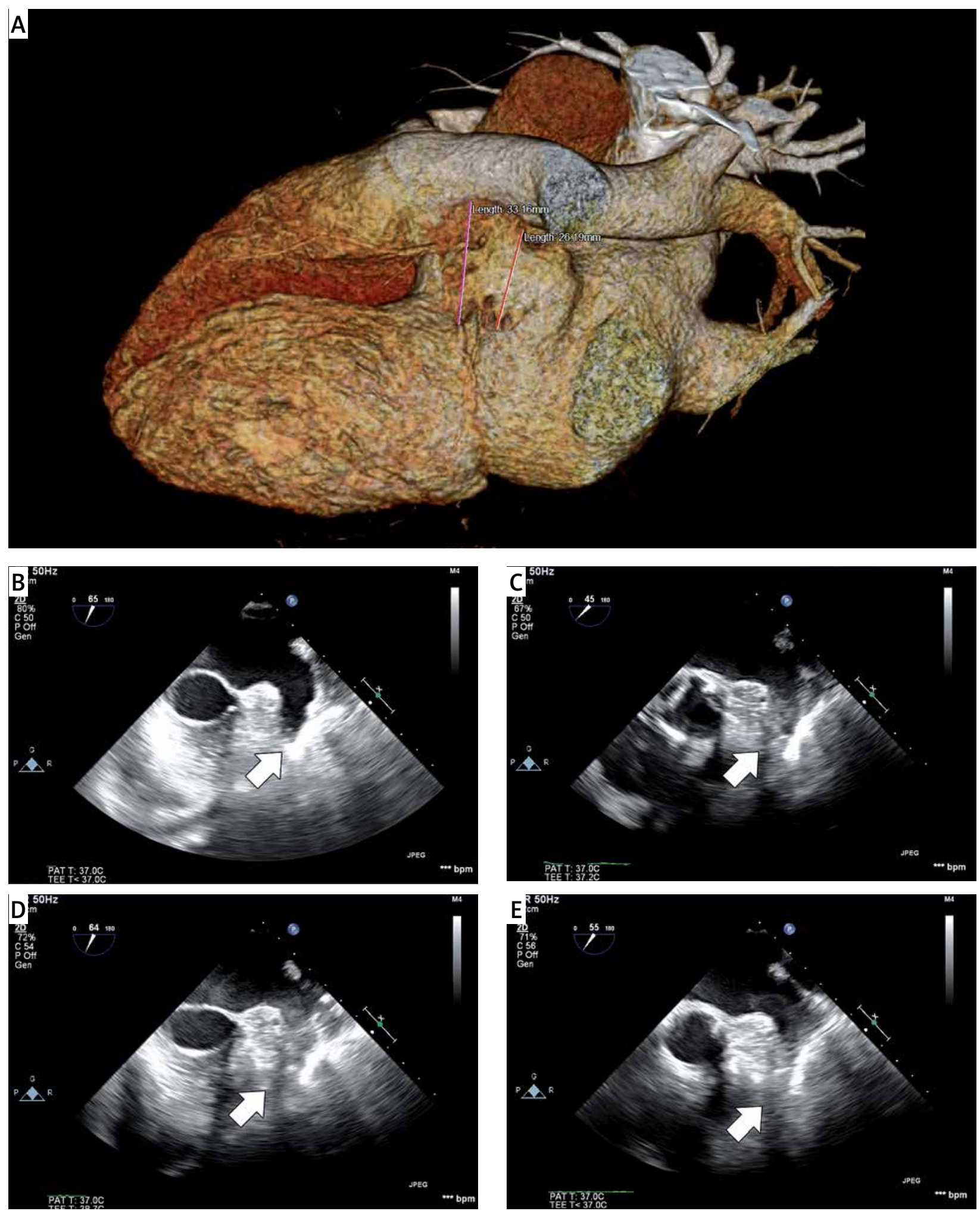

Figure 1. A - 3D computed tomography reconstruction scan with measurements of the left atrial appendage (LAA), B - transesophageal echocardiogram (TEE) at 3.57 PM with no thrombus in LAA (arrow), C - TEE at 4.27 PM with a fresh thrombus formed in less than 20 min, D - TEE at 4.31 PM with a solid fresh thrombus in the LAA, E - TEE the next day with partial dilution of the LAA thrombus 
ment. In such a case, epicardial LAA closure should be considered for effective thromboembolic protection.

\section{Funding sources}

Research grant No. UMO-2014/13/D/NZ5/01351 funded by the National Science Centre in Poland.

\section{Conflict of interest}

K. Bartus is a consultant to SentreHEART, Inc. R. Lee is a consultant and equity ownership for SentreHEART, Inc. Remaining authors declare no conflict of interest.

\section{References}

1. Bartus K, Podolec J, Lee RJ, et al. Atrial natriuretic peptide and brain natriuretic peptide changes after epicardial percutaneous left atrial appendage suture ligation using LARIAT device. J Physiol Pharmacol 2017; 68: 117-23.

2. Grygier M, Olasińska-Wiśniewska A, Araszkiewicz A, et al. The Watchman FLX - a new device for left atrial appendage occlusion - design, potential benefits and first clinical experience. Adv Interv Cardiol 2017; 13: 62-6.

3. Bartus K, Gafoor S, Tschopp D, et al. Left atrial appendage ligation with the next generation LARIAT(+) suture delivery device: Early clinical experience. Int J Cardiol 2016; 215: 244-7.

4. Litwinowicz R, Bartus $M$, Ceranowicz P, et al. Left atrial appendage occlusion for stroke prevention in diabetes mellitus patients with atrial fibrillation: long-term results. J Diabetes 2018 Jul 12 [Epub ahead of print]; doi: 10.1111/1753-0407.12824.

5. Litwinowicz R, Bartus M, Ceranowicz P, et al. Stroke risk reduction after left atrial appendage occlusion in elderly patients with atrial fibrillation: long-term results. Pol Arch Intern Med 2018; 128: 327-9. 